\title{
Conference
}

\section{Measurement of three independent components in impedance sensors using a single square wave}

\author{
Javier Lario-García, Ramon Pallàs-Areny* \\ Electronic Engineering Department, Technical University of Catalonia (UPC), Escola Politècnica Superior de Castelldefels (EPSC), \\ Avda. del Canal Olímpic s/ $n$, Castelldefels, Barcelona 08860, Spain
}

Received 31 July 2003; received in revised form 31 July 2003; accepted 15 August 2003

\begin{abstract}
Two-wire impedance-based sensors involving electrolytes add the impedance of the electrodes to the electrical impedance of the medium to measure. An equivalent circuit for the measured impedance is a resistance in series with the parallel combination of another resistance and a capacitance. If the two electrodes are modelled by equal impedances, the equivalent circuit for the complete set up consists of three impedance components, which can be determined from three independent measurements. This paper describes a novel method to obtain those three components using a single square wave voltage (period $2 T$ ) instead of several sine waves and provides the equations to calculate their value from the three current intensity amplitudes measured at $T / 8,3 T / 8$ and $5 T / 8$. Other measurement times would need different equations, but the same procedure applies. Anyway, the proposed method keeps the advantages of synchronous detection and relies on analytical solutions instead of the customary curve fitting procedures. Computer simulation and experimental results obtained by measuring the conductivity of known electrolyte samples validate the proposed method.
\end{abstract}

(C) 2003 Published by Elsevier B.V.

Keywords: Conductivity sensors; Time-domain measurement; Synchronous sampling

\section{Introduction}

Electrical impedance-based sensors rely on the impedance change produced by the measured quantity in a material or in the geometry of a circuit. The sensor is often modelled by several (lumped) circuit components and the measured quantity yields specific changes in one or more of those parameters ([1], Chapter 4). Simple models include a single circuit element, such as a resistance or a capacitance. More advanced models include a series or parallel combination of resistance and capacitance (Fig. 1a and b), and even three components, such as a resistance in series with the parallel combination of a resistance and capacitance (Fig. 1c).

Impedances modelled by several lumped elements can be measured in the frequency and in the time domains. Frequency domain measurements are based on impedance spectroscopy where several sine waves (voltage or current) are applied and the resulting current or voltage is measured ([2], Chapter 1). A model in then fitted to the experimental data. Time-domain measurement techniques usually apply a step voltage [3] or a bi-phasic voltage pulse [4], and measure

\footnotetext{
* Corresponding author. Tel.: +34-93-413-7096; fax: +34-93-413-7007. E-mail address: elerpa@eel.upc.es (R. Pallàs-Areny).
}

the current by converting it into a voltage and using an A/D converter, then a model is fitted to the FFT of the measured data. These time domain approaches need a fast A/D converter in order to have enough data points to perform the FFT and also a powerful enough data processor.

This paper describes a novel method to analytically determine three independent components of the impedance of a sensor by applying a single square-wave voltage and measuring the resulting current intensity at only three different selected times. The impedance model is assumed to be known in advance, then the response of this model to a square wave is derived, and the lumped impedance elements are calculated from the three currents measured. This procedure relies on basic calculus, the electronic circuitry is substantially easier to build and the computations are faster than in other time-domain techniques.

\section{Equivalent circuits in impedance-based sensors}

Measuring impedance by using sine waves is not convenient in digital instruments because of the need to generate accurate sine voltages or currents. Relaxation oscillators have been applied to the measurement of single capacitance ([1], Section 8.3), differential capacitance [5], and 


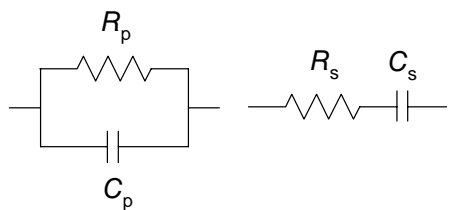

(a)

(b)

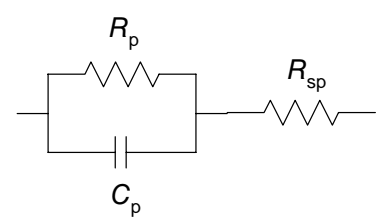

(c)

Fig. 1. Advanced models for impedance sensors include two or three independent components rather than a single element.

two-independent impedance components [6]. Some sensors, however, are better modelled by three independent impedance components rather than two independent components as shown in [7]. This is often the case when measuring the conductivity of electrolytes: electrode impedance can be modelled as shown in Fig. 1c [2], electrode leads have some resistance, and the electrolyte behaves as a resistance. Because measuring in an electrolyte involves electrodes, the equivalent circuit is that in Fig. 2 , where $R_{x}$ is the resistance of the electrolyte we wish to determine. Using a four-wire (or Kelvin) measurement set up overcomes the effect of electrode impedance and lead resistance ([1], Section 3.1 ), but conductivity probes with four electrodes are more difficult to manufacture, and hence more expensive. Furthermore, four-electrode methods need differential voltage amplifiers, which adds complexity to circuit design because of the need for a bias path different from the electrodes.

If the two electrodes in Fig. 2 have the same characteristics, the equivalent circuit reduces to $\left(2 R_{\mathrm{W}}+2 R_{\text {spe }}+R_{x}\right)$ in series with $\left(2 R_{\mathrm{pe}} \| C_{\mathrm{pe}} / 2\right)$ as in Fig. 1c. Determining those three components would enable us to calculate $R_{\mathrm{sp}}$ and then $R_{x}$ by first calibrating the system by measuring a solution of known conductivity. Here we propose to apply a single square-wave voltage and to measure the resulting current intensity at three different times (Fig. 3).

\section{Circuit analysis}

The equivalent impedance connected to the voltage source in Fig. 3 is

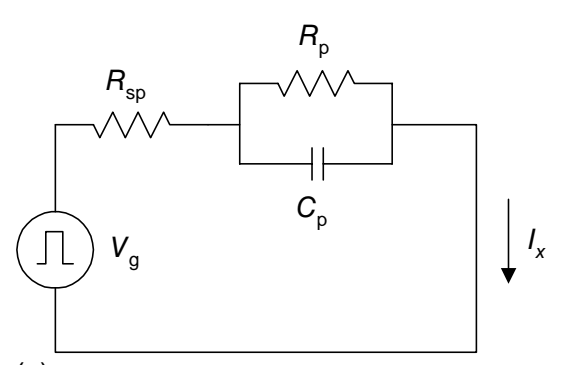

(a)

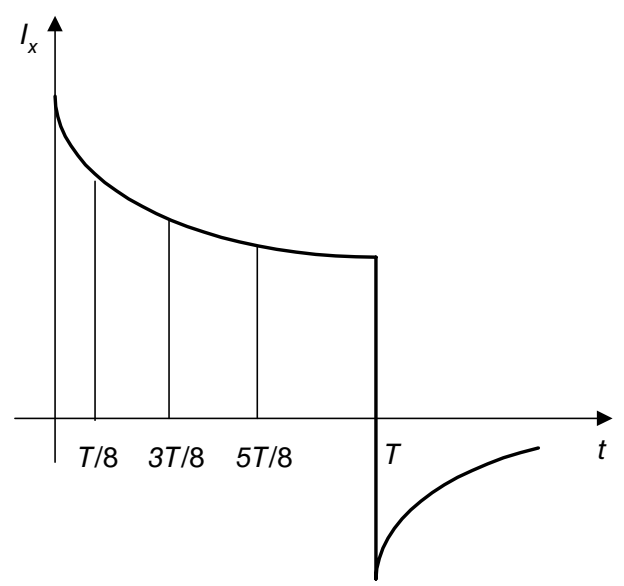

(b)

Fig. 3. The proposed method to measure three impedance components is based on (a) applying a square voltage and (b) measuring the resulting current intensity at three given times.

$Z(s)=R_{\mathrm{sp}} \frac{s+1 / \tau}{s+1 /\left(R_{\mathrm{p}} C_{\mathrm{p}}\right)}$

where

$\tau=R_{\mathrm{T}} C_{\mathrm{p}}$

and

$R_{\mathrm{T}}=\frac{R_{\mathrm{sp}} R_{\mathrm{p}}}{R_{\mathrm{sp}}+R_{\mathrm{p}}}$

When applying a voltage with square waveform, amplitude $V_{0}$, and period $2 T$, the resulting current intensity at any time instant within any positive half-period, $2 n T<t<$ $(2 n+1) T$ ( $n$ being any integer), can be calculated from the inverse Laplace transform of $I(s)=V(s) / Z(s)$. The result is

$I_{x}(t)=\frac{V_{0}}{R_{\mathrm{sp}}+R_{\mathrm{p}}}\left[2 \frac{R_{\mathrm{p}}}{R_{\mathrm{sp}}} \mathrm{e}^{-t / \tau} \frac{\mathrm{e}^{T / \tau}}{1+\mathrm{e}^{T / \tau}}+1\right]$

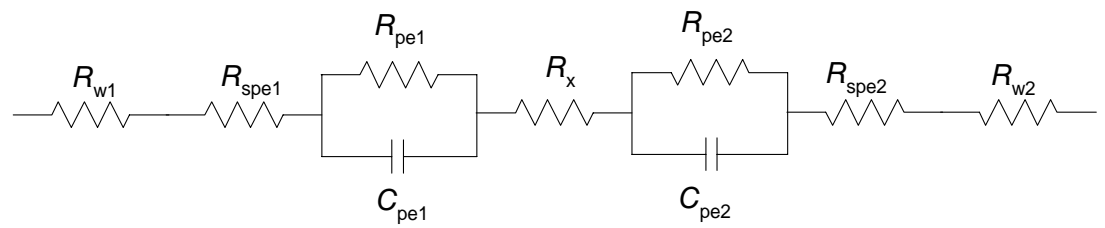

Fig. 2. Equivalent circuit for two electrodes and their leads $\left(R_{\mathrm{w} 1}, R_{\mathrm{w} 2}\right)$ immersed in an electrolyte whose conductivity $\left(1 / R_{x}\right)$ is the measured quantity. 
where, for a given impedance network, all the factors are constant exception made of the exponential factor that includes $t$. Therefore, measuring $I_{x}$ at three given instants $t_{1}$, $t_{2}$, and $t_{3}$, yields three equations that can be solved for the three unknowns $R_{\mathrm{sp}}, R_{\mathrm{p}}$, and $C_{\mathrm{p}}$. If those instants are chosen to be specific fractions of the period, this synchronous sampling reduces the noise-equivalent bandwidth and enhances interference rejection [8]. Those measurement times should be selected apart enough in order not to require a very-high-resolution current meter. If, for example, we select the instants shown in Fig. $3 \mathrm{~b}$ and name

$\Delta T=\frac{T}{8}$

the current at any instant $m \Delta T(0 \leq m<8$ ) is (see Appendix A)

$I_{x}(m \Delta T)=I_{\mathrm{F}}\left[2 A \mathrm{e}^{-m \Delta T \tau} \frac{\mathrm{e}^{T / \tau}}{1+\mathrm{e}^{T / \tau}}+1\right]$

where

$I_{\mathrm{F}}=\frac{V_{0}}{R_{\mathrm{sp}}+R_{\mathrm{p}}}$

is the intensity of the Faradic current (dc current) and

$A=R_{\mathrm{p}} / R_{\mathrm{sp}}$

Measuring at $t_{1}=T / 8, t_{2}=3 T / 8$, and $t_{3}=5 T / 8$, as shown in Fig. 3b, yields an equation system involving the measured currents $I_{x}(\Delta T), I_{x}(3 \Delta T)$, and $I_{x}(5 \Delta T)$. Solving that system yields

$$
\begin{aligned}
& \mathrm{e}^{\Delta T / \tau}=+\sqrt{\frac{I_{x}(3 \Delta T)-I_{x}(\Delta T)}{I_{x}(5 \Delta T)-I_{x}(3 \Delta T)}} \\
& A=\frac{I_{x}(3 \Delta T)-I_{x}(\Delta T)}{2\left[\mathrm{e}^{-3 \Delta T / \tau} I_{x}(\Delta T)-\mathrm{e}^{-\Delta T / \tau} I_{x}(3 \Delta T)\right] \frac{\mathrm{e}^{T / \tau}}{1+\mathrm{e}^{T / \tau}}} \\
& I_{\mathrm{F}}=\frac{I_{x}(3 \Delta T)}{2 A \mathrm{e}^{-3 \Delta T / \tau} \frac{\mathrm{e}^{T / \tau}}{1+\mathrm{e}^{T / \tau}}+1}
\end{aligned}
$$

In these two last equations $\mathrm{e}^{T / \tau}=\left(\mathrm{e}^{\Delta^{T / \tau}}\right)^{8}$ because $T=$ $8 \Delta T$ (Eq. (5)). Replacing (10) and (11) in (7) and (8), and solving for the resistances in Fig. 3a yields

$R_{\mathrm{sp}}=\frac{V_{0}}{I_{\mathrm{F}}(1+A)}$

$R_{\mathrm{p}}=A R_{\mathrm{sp}}$

From $R_{\mathrm{sp}}$ and $R_{\mathrm{p}}$, we can calculate $R_{\mathrm{T}}$ in (3), and from (2) and (9) the unknown capacitance is

$C_{\mathrm{p}}=\frac{\Delta T}{R_{\mathrm{T}} \ln \left(\mathrm{e}^{\Delta T / \tau}\right)}$

If the current intensities measured at $\Delta T, 3 \Delta T$ and $5 \Delta T$ were too close, the same procedure above for $m$ values other than 1,3 and 5 would lead to a similar equation system, yet different equations to replace (9) to (11). Hence, it is not a simple matter of replacing 1,3 and 5 by the new $m$ values, but the procedure is the same.

\section{Experimental results and discussion}

The proposed measurement method has been implemented by a custom circuit (square wave impedance meter (SWIM)) built on an add-on PC board. The current intensity $I_{x}$ is determined by sampling the drop in voltage across a known resistor at those specific times shown in Fig. $3 b$.

The method has been first validated by simulating and then experimentally measuring an impedance network like that in Fig. 1c built from $100 \Omega\left(R_{\mathrm{sp}}\right)$ in series with $1 \mu \mathrm{F} \| 1 \mathrm{k} \Omega$. Simulation with PSpice V6.0 (Microsim) yields the exact values for each of the three components.

The actual value of each network component has been determined by separately measuring each of them at $350 \mathrm{~Hz}$ using an Agilent impedance analyser that applies sine waves to the impedance under test. Its uncertainty for those component values and measurement frequency is about $0.3 \%$. Table 1 shows that the resulting errors for each component value when determined by applying the proposed method (i.e. a $350 \mathrm{~Hz}$ square wave) to the impedance network were $3.5 \%$ for $R_{\mathrm{sp}}, 0.2 \%$ for $R_{\mathrm{p}}$, and $0.3 \%$ for $C_{\mathrm{p}}$.

The impedance analyser detects that the capacitor $C_{\mathrm{p}}$ has a non-negligible equivalent parallel resistance (leakage resistance). This resistance will reduce the equivalent value of the resistor $R_{\mathrm{p}}$ in parallel with the capacitance in Fig. 1c. The relatively large error $(3.5 \%)$ for $R_{\mathrm{sp}}$ when using the proposed method (SWIM), can be in part attributed to the varying impedance of $C_{\mathrm{p}}$ for the range of frequencies involved in the square wave [9], to the series resistance of the capacitor, that the commercial impedance analyser cannot detect because of its inability to directly determine three independent components of an impedance network [10], to the finite aperture time of the sample and hold $(\mathrm{S} \& \mathrm{H})$ amplifier that samples the three voltages (proportional to $I_{x}$ ) in the proposed method, and to the uncertainty in $T$ and the three measurement instants. Anyway, those errors are acceptable for a broad range of conductivity sensors.

Table 1

Results when measuring an impedance network built from three passive components, whose value has been (separately) determined by a commercial impedance analyzer (Agilent 4294A)

\begin{tabular}{lcrl}
\hline $\begin{array}{l}\text { Impedance } \\
\text { network (Fig. 1c) }\end{array}$ & Agilent 4294A & SWIM & $\begin{array}{l}\text { Percentage } \\
\text { error }\end{array}$ \\
\hline$R_{\mathrm{sp}}: 100 \Omega(0.1 \%)$ & $100.05 \pm 0.01 \Omega$ & $103.6 \pm 0.1 \Omega$ & 3.5 \\
$R_{\mathrm{p}}: 1 \mathrm{k} \Omega(0.1 \%)$ & $1000.5 \pm 0.1 \Omega$ & $991.2 \pm 0.1 \Omega$ & 0.2 \\
$C_{\mathrm{p}}: 1 \mu \mathrm{F}(10 \%)$ & $(1.033 \mu \mathrm{F} \pm 1 \mathrm{nF}) / /$ & $1.036 \mu \mathrm{F} \pm 5 \mathrm{nF}$ & 0.3 \\
& $148 \mathrm{k} \Omega$ & & \\
\hline
\end{tabular}

The proposed method (SWIM) determines the three components from a single voltage injection. 
The proposed method has been applied to 15 samples whose conductivity, from 12.0 to $215800 \mu \mathrm{S}$ has been determined by a Knick meter (Konductometer 703) using a four-wire conductivity probe (ZU6985) that relies on the impedance model in Fig. 1b. The overall measurement error for the meter and the probe is less than $1 \%$. We have used two-wire platinum probes (Crison Instruments Ref. 52-92) connected to our circuit (SWIM). In order to better appreciate the efficacy and interest of the proposed method for three-component models for the electrode-electrolyte impedance, we first considered one model with two series impedance components (Fig. 1b) and then a model with three impedance components (Fig. 1c). Final results for the impedance model for the electrolyte are obtained by subtracting the series resistance of electrode leads and electrodes ( $R_{\mathrm{w} 1}+R_{\mathrm{w} 2}+2 R_{\mathrm{spe}}$ in Fig. 2$)$ from the readings obtained by SWIM. That series resistance of electrode leads and electrodes is measured by shorting the electrodes of the platinum cell and measuring the total resistance with an ohmmeter. Its value for the two-wire platinum probe used is $0.2 \Omega$.

Table 2 lists the measured conductivity values and Fig. 4 shows the corresponding errors. The error for low conductivities is small even for the two-component model. For conductivities above $40 \mathrm{mS}$, however, the error rapidly increases for the two-component model but remains small for the three-component model. This means that double-layer effects at the electrode-electrolyte interface ([2], Section 2.1) are negligible for low-conductivity electrolytes but be-
Table 2

Conductivity of 15 electrolyte samples determined by a four-wire Knick conductivity probe (model ZU6985) connected to a Knick conductivity meter (model Konductometer 703) and a two-wire probe (Crison Instruments Ref. 52-92) connected to the SWIM when considering a series two-component impedance model (Fig. 1b) and a three-component impedance model (Fig. 1c)

\begin{tabular}{rccc}
\hline Sample & \multicolumn{2}{l}{ Conductivity } & \\
\cline { 2 - 4 } & Knick & Model & Model \\
& $(\mu S) \pm 1 \%$ & Fig. 1b $(\mu S)$ & Fig. 1c $(\mu S)$ \\
\hline 1 & 12.0 & 12.0 & 12.1 \\
2 & 67.3 & 67.1 & 67.3 \\
3 & 114.1 & 113.7 & 114.0 \\
4 & 151.7 & 151.5 & 151.7 \\
5 & 286.5 & 285.8 & 286.6 \\
6 & 538.3 & 540.8 & 542.8 \\
7 & 1225 & 1206 & 1213 \\
8 & 2298 & 2268 & 2285 \\
9 & 5244 & 5132 & 5195 \\
10 & 11960 & 11730 & 11960 \\
11 & 22260 & 21600 & 22250 \\
12 & 45080 & 42740 & 44790 \\
13 & 100900 & 92030 & 99240 \\
14 & 155600 & 139070 & 153050 \\
15 & 215800 & 188300 & 211200 \\
\hline
\end{tabular}

come very important for high-conductivity electrolytes. A series two-component impedance model (Fig. 1b) underestimates high conductivities. The three-component impedance model (Fig. 1c) better approximates the actual impedance

\section{Conductivity ( $\mu S)$}

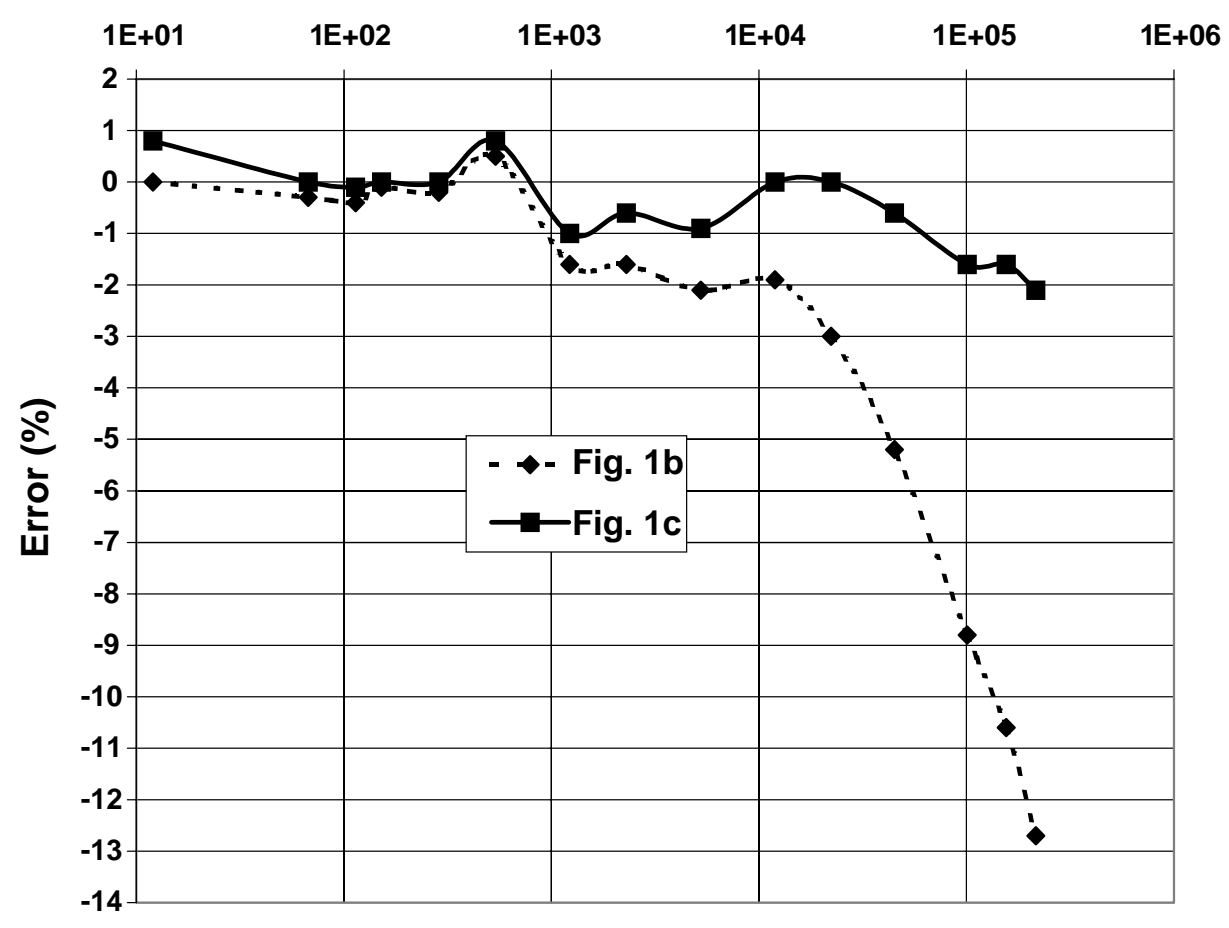

Fig. 4. Conductivity error for the electrolyte samples in Table 2 when applying the proposed method. 
of the system, and the proposed measurement method accurately determines those three components by applying a single voltage with a two-wire probe.

\section{Conclusions}

Electrolyte conductivity sensors and other impedance sensors are better modelled by an impedance network that includes three independent components rather than a single impedance component. Impedance spectroscopy permits the determination of those three components but requires the injection of several sine waves and solving the resulting equation system. Using a single square wave voltage also permits the determination of those components if the current intensity across the sample is measured at three given times and the corresponding equation system is solved.

This method has been tested by measuring both a network built from known passive components and the conductivity of known electrolyte samples. The small errors obtained validate the proposed method, which is particularly advantageous when measuring samples whose conductivity exceeds $40 \mu \mathrm{S}$. The same technique can be applied to other impedance-based sensors or measurement techniques involving electrodes and electrolytes provided that they can be modelled by the network in Fig. 1c.

\section{Acknowledgements}

This work has been funded in part by the Spanish Ministry of Science and Technology, project TAP99-0742.

\section{Appendix A}

The intensity of the current through an impedance $Z(s)$ when injecting a voltage whose Laplace transform is $V(s)$, is

$I(s)=\frac{V(s)}{Z(s)}$

A method to obtain the inverse Laplace transform in order to determine $i(t)$ is to decompose the square waveform of amplitude $V_{0}$ and period $2 T$ in a sum of step signals delayed by $T$ from each other (Fig. A.1). For a linear system, the current can be obtained by adding the current produced by each step voltage input.

The impedance of the network in Fig. 1c is

$$
Z(s)=R_{\mathrm{sp}} \frac{s+\left(\left(R_{\mathrm{sp}}+R_{\mathrm{p}}\right) /\left(R_{\mathrm{sp}} R_{\mathrm{p}} C_{\mathrm{p}}\right)\right)}{s+\left(1 / R_{\mathrm{p}} C_{\mathrm{p}}\right)}=R_{\mathrm{sp}} \frac{s+(1 / \tau)}{s+\left(1 / \tau_{1}\right)}
$$

where

$$
R_{\mathrm{T}}=\frac{R_{\mathrm{sp}} R_{\mathrm{p}}}{R_{\mathrm{sp}}+R_{\mathrm{p}}}
$$
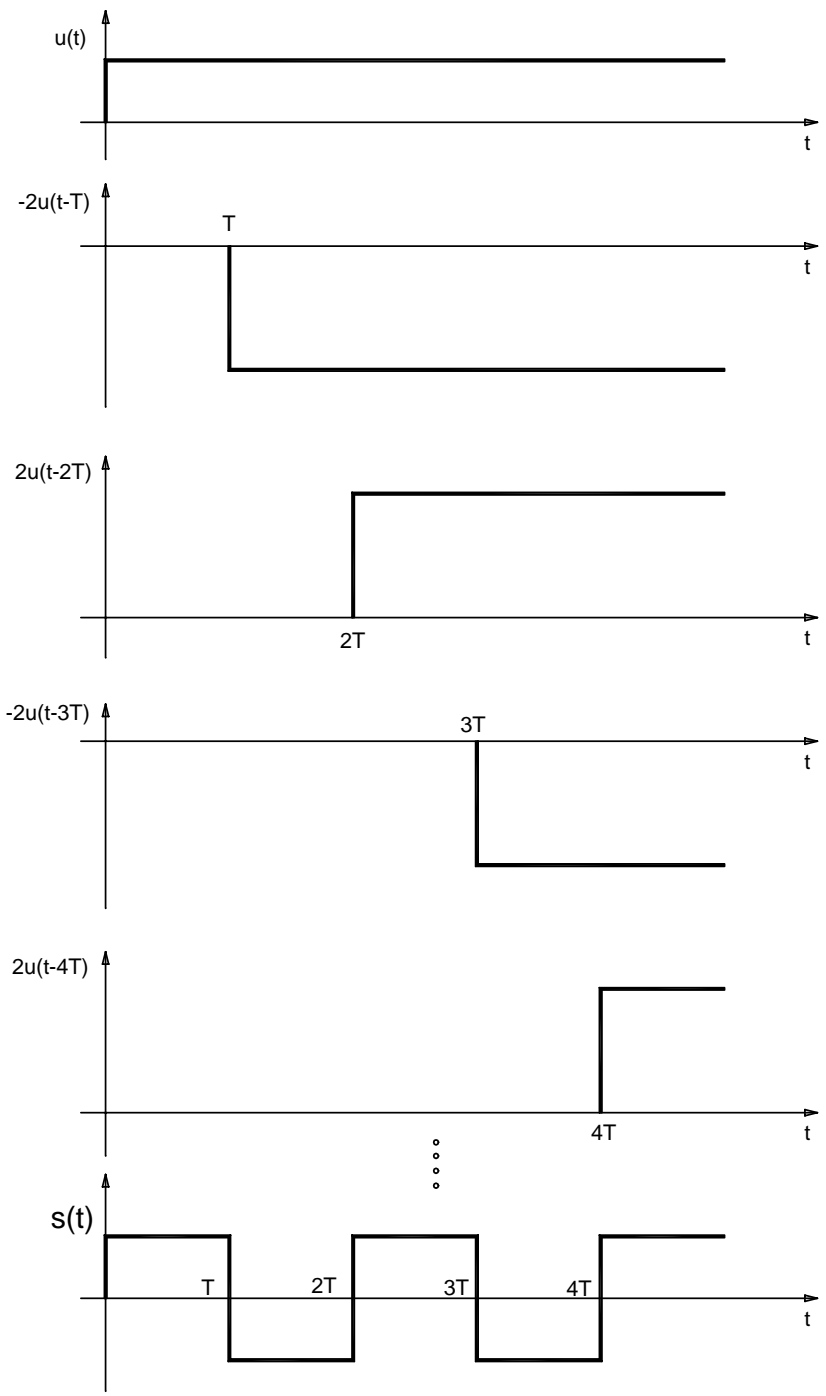

Fig. A.1. Any square wave voltage $s(t)$ can be decomposed as a sum of step voltages with amplitudes $+1,-2,+2,-2,+2$, and so on, each step delayed by $N T$ seconds $(N=0,1,2, \ldots)$.

and

$\tau=R_{\mathrm{T}} C_{\mathrm{p}}$

$\tau_{1}=R_{\mathrm{p}} C_{\mathrm{p}}$

The square wave voltage can be decomposed into (Fig. A.1)

$$
\begin{aligned}
u(t)= & V_{0} \operatorname{sign}(\cos (2 \pi f t)) \\
= & V_{0}[u(t)-2 u(t-T)+2 u(t-2 T) \\
& -2 u(t-3 T)+\cdots]
\end{aligned}
$$

where $u(t)$ is the unit step and $f=1 /(2 T)$ is the signal frequency.

A single step voltage of amplitude $V_{0} u(t)$, whose transform is

$V(s)=\frac{V_{0}}{s}$ 
yields a current

$$
\begin{aligned}
I_{\mathrm{U}}(s) & =\frac{V(s)}{Z(s)}=\frac{V_{0}}{R_{\mathrm{sp}}} \frac{1}{s}\left[\frac{s+\left(1 / \tau_{1}\right)}{s+(1 / \tau)}\right] \\
& =\frac{V_{0}}{R_{\mathrm{sp}}} \frac{1}{s}\left[\frac{s}{s+(1 / \tau)}\right]+\frac{V_{0}}{R_{\mathrm{sp}}} \frac{1}{s}\left[\frac{1 / \tau_{1}}{s+(1 / \tau)}\right]
\end{aligned}
$$

that can be written as the sum of simple fractions,

$I_{\mathrm{U}}(s)=\frac{A}{s}+\frac{B}{s+(1 / \tau)}+\frac{C}{s+(1 / \tau)}$

The corresponding inverse transform is

$i_{\mathrm{U}}(t)=L^{-1}\{I(s)\}=\frac{V_{0}}{R_{\mathrm{sp}}}\left[\mathrm{e}^{-t / \tau}+\frac{\tau}{\tau_{1}}-\frac{\tau}{\tau_{1}} \mathrm{e}^{-t / \tau}\right]$

From (A.4) and (A.5) we have

$$
\frac{\tau}{\tau_{1}}=\frac{C_{\mathrm{p}} R_{\mathrm{sp}} R_{\mathrm{p}} /\left(R_{\mathrm{sp}}+R_{\mathrm{p}}\right)}{C_{\mathrm{p}} R_{\mathrm{p}}}=\frac{R_{\mathrm{sp}}}{R_{\mathrm{sp}}+R_{\mathrm{p}}}
$$

Therefore (A.10) is equivalent to

$i_{\mathrm{U}}(t)=\frac{V_{0}}{R_{\mathrm{sp}}+R_{\mathrm{p}}}\left[\frac{R_{\mathrm{p}}}{R_{\mathrm{sp}}} \mathrm{e}^{-t / \tau}+1\right]=I_{\mathrm{F}}\left[A \mathrm{e}^{-t / \tau}+1\right]$

where $I_{\mathrm{F}}$ is the Faradic current

$I_{\mathrm{F}}=\frac{V_{0}}{R_{\mathrm{sp}}+R_{\mathrm{p}}}$

and

$A=\frac{R_{\mathrm{p}}}{R_{\mathrm{sp}}}$

The current produced by the square wave voltage in (A.6) is then

$$
\begin{aligned}
i(t)= & i_{\mathrm{U}}(t)-2 i_{\mathrm{U}}(t-T)+2 i_{\mathrm{U}}(t-2 T) \\
& -2 i_{\mathrm{U}}(t-3 T)+\cdots
\end{aligned}
$$

At any given time instant, the current intensity will be the result of the currents produced by the previous step voltages. For $t$ such that $2 M T \leq t<(2 M+1) T, M$ being any positive integer, we can consider $t=2 M T+m \Delta T$, where $\Delta T=$ $T / m$, and $m$ is any positive integer larger than 2 . The current at time $t$ is then

$$
\begin{aligned}
i(2 M T+m \Delta T)= & i_{\mathrm{U}}(2 M T+m \Delta T) \\
& -2 \sum_{k=1}^{M} i_{\mathrm{U}}((2(M-k)+1) T+m \Delta T) \\
& +2 \sum_{n=1}^{M} i_{\mathrm{U}}(2(M-n) T+m \Delta T)
\end{aligned}
$$

The first term is the current produced by the first voltage step of amplitude $V_{0}$. The second term is the sum of the current produced by all the voltage steps with amplitude
$-2 V_{0}$ (at $T, 3 T$, and so on). The third term is the sum of the current produced by all the voltage steps with amplitude $+2 V_{0}$. From (A.12), when $M \gg 1$, each term of (A.16) can be, respectively, written as

$$
i_{\mathrm{U}}(2 M T+m \Delta T)=I_{\mathrm{F}}
$$

$$
\begin{aligned}
& -2 \sum_{k=1}^{M} i_{\mathrm{U}}((2(M-k)+1) T+m \Delta T) \\
& \quad=2 I_{\mathrm{F}}\left[A \mathrm{e}^{-m \Delta T / \tau}\left(\frac{\mathrm{e}^{T / \tau}}{1-\mathrm{e}^{2 T / \tau}}\right)+(M-1)\right]
\end{aligned}
$$

$$
\begin{aligned}
& 2 \sum_{n=1}^{M} i_{\mathrm{U}}(2(M-n) T+m \Delta T) \\
& \quad=-2 I_{\mathrm{F}}\left[A \mathrm{e}^{-m \Delta T / \tau}\left(\frac{\mathrm{e}^{2 T / \tau}}{1-\mathrm{e}^{2 T / \tau}}\right)+(M-1)\right]
\end{aligned}
$$

Substituting these expressions in (A.16) yields

$$
\begin{aligned}
& i(2 M T+m \Delta T) \\
& =I_{\mathrm{F}}-2 I_{\mathrm{F}}\left[A \mathrm{e}^{-m \Delta T / \tau}\left(\frac{\mathrm{e}^{T / \tau}}{1-\mathrm{e}^{2 T / \tau}}\right)+(M-1)\right] \\
& +2 I_{\mathrm{F}}\left[A \mathrm{e}^{-m \Delta T / \tau}\left(\frac{\mathrm{e}^{2 T / \tau}}{1-\mathrm{e}^{2 T / \tau}}\right)+(M-1)\right]
\end{aligned}
$$

This expression can be simplified to finally obtain that the current through the impedance network in Fig. 1c is

$i(2 M T+m \Delta T)=I_{\mathrm{F}}\left[2 A \mathrm{e}^{-m \Delta T / \tau} \frac{e^{T / \tau}}{1+e^{T / \tau}}+1\right]$

which is Eq. (6) in the text.

\section{References}

[1] R. Pallàs-Areny, J.G. Webster, Sensors and Signal Conditioning, second ed., Wiley, New York, 2001.

[2] J. Ross Macdonald (Ed.), Impedance Spectroscopy Emphasizing Solid Materials and Systems, Wiley, New York, 1987.

[3] C.E.B. Neves, M.N. Souza, A method for bio-electrical impedance analysis based on a step-voltage response, Physiol. Meas. 21 (2000) 395-408.

[4] A.R. Waterworth, P. Milnes, R.H. Smallwood, B.H. Brown, Cole equation modelling to measurements made using an impulse driven transfer impedance system, Physiol. Meas. 21 (2000) 137-144.

[5] K. Mochizuki, K. Watanabe, T. Masuda, M. Katsura, A relaxation-oscillator-based interface for high-accuracy ratiometric signal processing of differential-capacitance transducers, IEEE Trans. Instrum. Meas. 47 (1998) 11-15.

[6] S.T. Nihtianov, G.C.M. Meijer, A novel technique to measure two independent components of impedance sensors with a simple relaxation oscillator, IEEE Trans. Instrum. Meas. 50 (2001) 1563-1567.

[7] P.T. Kissinger, W.R. Heineman (Eds.), Laboratory Techniques in Electroanalytical Chemistry, second ed., Marcel Dekker, New York, 1996, pp. 237-265.

[8] R. Pallàs-Areny, O. Casas, A novel differential synchronous demodulator for AC signals, IEEE Trans. Instrum. Meas. 45 (1996) 413-416. 
[9] N. Hagiwara, T. Saegusa, An RC discharge digital capacitance meter, IEEE Trans. Instrum. Meas. 32 (1983) 316-321.

[10] H. Haruta, Agilent Technologies Impedance Measurement Handbook, second ed., Agilent Technologies, 2000, pp. 1-1-1-7.

\section{Biographies}

Javier Lario-García received the Telecommunication Engineer degree from the Technical University of Catalonia (UPC), Barcelona, Spain, in 1995. He has 8 years experience in industrial design of medical and analytical instrumentation. He is currently a PhD candidate at the Department of Electronic Engineering at the same University. His research work is about electrical impedance analysis based on digital signals, particularly applied to conductivity measurements in electrolytes and bioimpedance.

Ramon Pallàs-Areny received the Ingeniero Industrial and Doctor Ingeniero Industrial degrees from the Technical University of Catalonia (UPC), Barcelona, Spain, in 1975 and 1982, respectively. He is a Pro- fessor of Electronic Engineering at the same University, and teaches courses in medical and electronic instrumentation. In 1989 and 1990 , he was a visiting Fulbright Scholar and, in 1997 and 1998, he was an Honorary Fellow at the University of Wisconsin, Madison. In 2001, he was nominated Professor Honoris Causa by the Faculty of Electrical Engineering of the University of Cluj-Napoca (Romania). His research includes instrumentation methods and sensors based on electrical impedance measurements, sensor interfaces, non-invasive physiological measurements and electromagnetic compatibility in electronic systems. $\mathrm{He}$ is the author of several books on instrumentation in Spanish and Catalan, the latest one being Sensors and Interfaces, Solved Problems (1999), published by Edicions UPC, Barcelona, Spain. He is also coauthor (with John G. Webster) of Sensors and Signal Conditioning, second ed. (Wiley, 2001, New York), and Analog Signal Processing (Wiley, 1999, New York).

Dr. Pallàs-Areny was a recipient, with John G. Webster, of the 1991 Andrew R. Chi Prize Paper Award from the Instrumentation and Measurement Society (IEEE). He is a Fellow of the IEEE and member of the International Society for Measurement and Control (ISA). 